\title{
A CRESCENTE IMPORTÂNCIA DAS UNIVERSIDADES E INSTITUTOS PÚBLICOS DE PESQUISA NO PROCESSO DE CATCHING-UP TECNOLÓGICO*
}

\section{Luciano Martins Costa Póvoa ${ }^{* *}$}

\begin{abstract}
RESUMO O objetivo deste artigo é analisar como as universidades e os institutos públicos de pesquisa podem contribuir para o avanço tecnológico de países em desenvolvimento e apresentar argumentos que sugerem que a importância desses agentes tem aumentado com os recentes paradigmas tecnológicos e o fortalecimento da proteção à propriedade intelectual no cenário internacional. Para tanto, são discutidas as relações entre ciência e tecnologia e as características presentes nas experiências de catching-up de sucesso.
\end{abstract}

Palavras-chave: catching-up; universidade; institutos públicos de pesquisa; tecnologia

Código JEL: O30; O31

\section{THE INCREASING IMPORTANCE OF UNIVERSITIES AND PUBLIC RESEARCH INSTITUTIONS IN THE CATCHING-UP PROCESS}

ABSTRACT The aim of this paper is to analyze the role of universities and public research institutions in the process of technical catch-up and to discuss how their importance have increased along the last century due to the new technological paradigms and international intellectual property rights rules. The relationship between science and technology is investigated as well as the common characteristics in the success experiences of catching-up.

Key words: catching-up; university; public research institutions; technology

\footnotetext{
* Artigo recebido em 21 de junho de 2007 e aprovado em 21 de agosto de 2008.

** Doutor em Economia pelo Cedeplar - UFMG. O autor agradece os comentários e as sugestões de Jorge Britto, Renato Garcia e Wilson Suzigan. Um agradecimento especial para Eduardo Albuquerque, Richard Nelson e para os pareceristas anônimos desta revista, que em muito contribuíram para o aperfeiçoamento deste artigo. $\mathrm{O}$ autor agradece ainda o apoio financeiro da Fapemig e Capes. Os erros que ainda fizerem parte deste trabalho são de responsabilidade exclusiva do autor, e-mail:povoa@cedeplar.ufmg.br
} 


\section{INTRODUÇÃO}

Uma empresa raramente inova de forma isolada, sem que haja a contribuição de outras organizações da economia e da sociedade. O desenvolvimento de um novo produto, ou um novo processo, por parte de uma empresa envolve muitas vezes a participação de fornecedores, informações provenientes de usuários, universidades, institutos de pesquisa e até mesmo de concorrentes.

Nas últimas décadas, tem sido cada vez mais destacada a interação entre a pesquisa acadêmica e a inovação industrial (Mansfield, 1995; Rosenberg e Nelson, 1994; Rosenberg, 2000). Estudos sobre experiências recentes de catching-up tecnológico, ou seja, a redução da lacuna de desenvolvimento tecnológico entre os países menos e mais avançados, sugerem que as pesquisas realizadas nas universidades e em institutos públicos de pesquisa podem gerar contribuições relevantes para o desenvolvimento econômico de um país, ajudando principalmente no acompanhamento e aprendizado de novas tecnologias (Albuquerque, 2001; Mazzoleni, 2003; Mazzoleni e Nelson, 2007).

O objetivo deste artigo é analisar como as universidades e os institutos públicos de pesquisa (IPP daqui para frente) podem contribuir para o avanço tecnológico de países em desenvolvimento e apresentar argumentos que sugerem que a importância desses agentes para o avanço tecnológico tem aumentado com os recentes paradigmas tecnológicos e o fortalecimento da proteção à propriedade intelectual no cenário internacional. A primeira seção deste artigo mostra o papel das universidades e IPP no sistema nacional de inovação, como se dá a interação com a indústria e como o papel das universidades pode mudar com o desenvolvimento econômico. Alguns elementos característicos de experiências históricas de catching-up são analisados na segunda seção, e na terceira seção é discutido o papel das universidades e IPP no processo de catching-up. Na quarta seção, os novos desafios a serem enfrentados pelos países que objetivam realizar o catching-up tecnológico são discutidos, bem como a importância das universidades e IPP nesse contexto. A quinta seção apresenta os comentários finais. 


\section{O PAPEL DAS UNIVERSIDADES E INSTITUTOS PÚBlicos DE PESQUISA EM UM SISTEMA NACIONAL DE INOVAÇÃO}

Um conceito importante para compreender o papel das universidades e dos IPP no avanço tecnológico é o de sistema nacional de inovação, que pode ser entendido como o conjunto de instituições, agentes e mecanismos de um país que contribuem para a criação, desenvolvimento e difusão das inovações tecnológicas. ${ }^{1}$ Entre essas instituições, agentes e mecanismos, destacam-se as empresas e seus laboratórios de pesquisa e desenvolvimento, o sistema educacional, as universidades, os institutos de pesquisa, a estrutura do sistema financeiro e as leis de propriedade intelectual. A importância do sistema nacional de inovação para o avanço tecnológico não está apenas na existência desse conjunto de organizações e instituições, mas, principalmente, de fortes interações entre os componentes do sistema de inovação que permitam uma atuação coerente. Como cada setor industrial tem suas particularidades e sua própria dinâmica, também é possível aplicar esse mesmo conceito para analisar os sistemas setoriais de inovação. ${ }^{2}$

A relação analisada neste artigo é a interação entre universidades/IPP e a indústria. Para entender essa relação, é importante destacar que o advento da tecnologia moderna, baseada na ciência, fez com que surgisse uma complexa relação entre ciência e tecnologia, conforme sugerem Nelson e Rosenberg (1993). Através de vários exemplos históricos, esses autores mostram que a ciência influencia o surgimento de inovações tecnológicas. Desse modo, as universidades, bem como os IPP, possuem um papel crucial em um sistema nacional de inovação. ${ }^{3}$ Esses agentes atuam como formadores de cientistas e engenheiros e como fontes de conhecimentos científicos e de pesquisas que fornecem técnicas úteis para o desenvolvimento tecnológico. A complexidade da relação entre ciência e tecnologia fica mais evidente ao se considerar que essa relação não possui apenas um sentido de causalidade. Segundo Nelson e Rosenberg, a tecnologia também exerce forte influência na pesquisa científica.

\subsection{A influência da tecnologia na ciência}

Vários estudos já apontaram a influência da tecnologia sobre a ciência, mas o trabalho de Rosenberg (1982) talvez tenha sido o que melhor destacou a essência econômica dessa relação. Rosenberg analisa as ligações entre ciên- 
cia e economia e discute com bastante clareza a questão de "quão exógena é a ciência", argumentando que a tecnologia influencia a atividade científica de várias formas. Para o autor, a tecnologia não pode ser pensada como a aplicação de um conhecimento científico já existente. Pelo contrário, “(...) the normal situation in the past, and to a considerable degree also in the present, is that technological knowledge has preceded scientific knowledge" (Rosenberg, 1982, p. 144). Muitos aprimoramentos tecnológicos ainda ocorrem pelo método de tentativa e erro, especialmente para solucionar questões para as quais ainda não há uma explicação científica. Embora Rosenberg não tenha destacado, é importante observar que mesmo o método de tentativa e erro é guiado atualmente por algum conhecimento científico prévio, de forma a restringir as possibilidades a serem tentadas (Moravcsik, 1983). Dado que os aprimoramentos tecnológicos são direcionados por uma lógica econômica, os conhecimentos científicos podem ser utilizados para delinear o campo de possibilidades de forma a tentar reduzir custos.

De acordo com Rosenberg (1982, p. 144), a tecnologia tanto tem servido como uma fonte de questões e problemas a serem abordados e resolvidos pela pesquisa científica como também acumula um conjunto de conhecimentos empíricos a serem organizados e avaliados pelos cientistas. $\mathrm{O}$ autor também destaca que o progresso tecnológico, ao apontar as direções onde novas pesquisas científicas poderão obter maiores retornos financeiros ou sociais, influencia de forma significativa a elaboração de uma agenda subseqüente para a ciência (p. 147-148). Uma inovação tecnológica pode influenciar até a pesquisa básica, como no caso da invenção do transistor em 1948, afetando substancialmente o desenvolvimento da física do estado sólido. Nesse caso, de acordo com o autor, "clearly, the main flow of scientific knowledge during this period was from industry to the university" (p. 155).

Por fim, Rosenberg aponta que um outro canal por onde os avanços tecnológicos podem afetar a pesquisa científica é através do desenvolvimento da instrumentação, ou seja, novos métodos de observar, testar e medir os fenômenos como, por exemplo, o raio X e o microscópio (p. 158).

Em sua interpretação, Rosenberg sugere que a ligação entre a economia e a ciência se faz, principalmente, através da tecnologia, que indica os rumos onde a pesquisa científica encontraria resultados financeiros potencialmente mais elevados. $\mathrm{O}$ autor conclui afirmando que: 
Powerful economic impulses are shaping, directing, and constraining the scientific enterprise. These impulses are rooted in two facts: First, scientific research is a costly activity; second, it can be directed in ways that may yield large economic rewards (...) I believe that the industrialization process inevitably transforms science into a more and more endogenous activity by increasing its dependence upon technology. Technological considerations, I have argued, are a major determinant of the allocation of scientific resources (Rosenberg, 1982, p. 159).

Essa existência de uma complexa relação interativa entre ciência e tecnologia faz com que o fluxo de conhecimentos entre a academia e a indústria não seja uma via de sentido único, tornando o estudo do papel da universidade e dos IPP um sistema nacional de inovação, e a sua interação com o setor industrial extremamente fecunda.

\subsection{A influência da pesquisa acadêmica nas empresas}

Mowery e Sampat (2005) apresentam um importante apanhado dos estudos sobre a interação universidade-indústria e a importância da pesquisa acadêmica para os avanços tecnológicos. Os autores apontam alguns "produtos" economicamente importantes resultantes da pesquisa acadêmica, tais como: informações tecnológicas e científicas; equipamentos e instrumentação; capital humano; redes de capacidade científica e tecnológica; e protótipos de novos produtos e processos. Destacam também que o fortalecimento da interação entre a universidade e as outras instituições e agentes do sistema nacional de inovação, em especial a indústria, é fundamental para que a primeira possa contribuir de forma mais eficaz para o avanço tecnológico. Para os autores, tem havido um fortalecimento dos laços entre a universidade e a indústria em muitos países da OCDE, e isso tem ocorrido devido a dois tipos de fatores: um externo e outro interno às universidades. O fator externo é a atuação cada vez mais comum dos governos de países industrializados e em desenvolvimento no sentido de utilizar as universidades na promoção do desenvolvimento na era da economia baseada no conhecimento. Uma conseqüência desse tipo de atuação governamental é a criação de parques científicos e tecnológicos em vários países, bem como tentativas de replicar as experiências do vale do silício e da rota 128 nos Estados Unidos. Já o fator interno diz respeito às crescentes restrições orça- 
mentárias que as universidades dos países da OCDE vêm enfrentando. As reduções dos recursos públicos por pesquisador em face do aumento dos custos das pesquisas de ponta têm feito as universidades adotarem uma postura mais agressiva e "empreendedora" na busca de novas fontes de recursos para a pesquisa (Mowery e Sampat, 2005, p. 211).

O aumento da cooperação entre universidade e indústria também é retratado por Meyer-Krahmer e Schmoch (1998) e Cohen et al. (2002), tomando, principalmente, a proporção crescente de pesquisas financiadas pela indústria. Na Alemanha, os fundos industriais para as atividades de pesquisa nas universidades aumentaram, em termos reais, 2,4 vezes entre 1985 e 1995 (Meyer-Krahmer e Schmoch, 1998, p. 835). Os autores estimam que os departamentos universitários mais relacionados com tecnologia, como as engenharias, recebem uma fatia em torno de $20 \%$ de seus fundos provenientes da indústria. No caso dos EUA, entre 1985 e 1998, os fundos industriais para a pesquisa universitária aumentaram de US\$ 630 milhões para US\$1,896 bilhão (em dólares de 2000), além de ter ocorrido um aumento de $60 \%$ dos centros de P\&D de indústrias em conjunto com universidades (Cohen et al., 2002, p. 3).

Com o objetivo de avaliar os "efeitos reais da pesquisa acadêmica", Jaffe (1989) estima uma "função de produção de conhecimento", tendo o número de patentes como variável dependente e os gastos privados e universitários com P\&D como variáveis independentes. O autor sugere que a atividade inovadora das firmas (medida pelas patentes) é influenciada pelos gastos universitários com pesquisa, sendo esse resultado um indício da presença de transbordamentos de conhecimento. O estudo de Jaffe, que estimulou vários outros trabalhos que buscaram analisar a presença de knowledge spillovers, propõe uma relação de causa e efeito entre a pesquisa acadêmica e o P\&D das empresas, gerando tecnologia, medida em patentes. Porém, não lança luz alguma sobre as relações existentes entre a pesquisa nas universidades e a inovação na indústria, bem como os canais de transferência desses conhecimentos.

Dos estudos que buscaram compreender com maior profundidade as contribuições da pesquisa acadêmica para a indústria, destacam-se o Yale Survey (Klevorick et al., 1995; Levin et al., 1987) e o Carnegie Mellon Survey (Cohen et al., 2002), ambos realizados nos EUA. O Yale Survey (YS), realiza- 
do em 1983-1984, teve como objetivo geral entender as estratégias de P\&D de empresas de vários setores industriais norte-americanos para o desenvolvimento de produtos e processos. Os questionários foram endereçados aos administradores responsáveis pelo P\&D das empresas. O estudo obteve resposta de 650 administradores, representantes de 130 setores industriais. Como resultado geral, observou-se que a contribuição da pesquisa acadêmica para o avanço tecnológico ocorre através de vários mecanismos e varia entre os setores industriais. Assim, existem ramos da ciência que são considerados mais relevantes para as inovações tecnológicas em alguns setores industriais do que em outros. Por exemplo, 99 setores industriais consideraram que ciência dos materiais foi relevante para o seu progresso tecnológico. No caso da geologia, apenas quatro setores industriais apontaram-na como uma ciência relevante para o seu progresso tecnológico recente.

Klevorick et al. (1995) apontam os avanços no conhecimento científico como sendo a fonte mais importante de oportunidades tecnológicas. ${ }^{4}$ A ciência fornece dados, explicações teóricas, técnicas e soluções gerais de problemas que podem ser utilizados na pesquisa e no desenvolvimento industrial, além de desenvolver conhecimentos que podem abrir diretamente novas possibilidades tecnológicas. Analisando as respostas dos questionários, os autores puderam identificar que o conjunto de conhecimentos já incorporado em um dado ramo científico é considerado mais importante para os avanços tecnológicos recentes do que a pesquisa acadêmica nesse mesmo ramo científico. Esse resultado deve ser interpretado com cautela, pois não significa que a pesquisa realizada nas universidades e IPP não seja importante para a indústria, e sim que a pesquisa acadêmica contemporânea em geral não afeta a indústria diretamente, porque os resultados das pesquisas podem ainda não estar consolidados e são de difícil monitoramento e assimilação por parte da indústria. Conforme destacam Gibbons e Johnston (1974, p. 241), embora os resultados de pesquisas científicas sejam publicados em periódicos, esse conhecimento não se torna um bem de livre acesso até se tornar parte do currículo acadêmico. Antes disso, só é diretamente acessível a poucos especialistas da área.

O Carnegie Mellon Survey (CMS), realizado em 1994 pelo mesmo grupo de pesquisadores do YS, pode ser entendido como uma continuação, mais do que uma atualização, do YS. A amostra foi constituída a partir de todas as 
unidades de P\&D de indústrias de manufaturas dos EUA, o que resultou em 3.240 laboratórios de P\&D. Os autores receberam 1.478 respostas (uma taxa de retorno de 46\%). O questionário buscou analisar a importância da pesquisa universitária e dos institutos públicos para a inovação industrial, enquanto o YS perguntou apenas sobre as universidades. ${ }^{5}$ Cohen et al. (2002) analisam a influência da pesquisa realizada nas universidades e laboratórios públicos (chamadas de pesquisa pública pelos autores) para a conclusão e sugestão de projetos de P\&D industrial. Os resultados mostram que os consumidores são considerados a principal fonte de informações para a sugestão de novos projetos (sendo indicados por 90,4\% dos respondentes), enquanto a conclusão de projetos existentes tem como principal fonte de informação a própria linha de operação da empresa (indicadas por 78,2\% dos respondentes). As universidades e laboratórios públicos possuem um papel modesto tanto na geração de novos projetos de P\&D industrial quanto na conclusão de projetos existentes, tendo sido o papel desses agentes considerado relativamente mais relevante na conclusão de projetos do que na sugestão de novos (indicados por 31,6\% e 36,3\% dos respondentes, respectivamente). ${ }^{6}$ Isso mostra a fragilidade do argumento do "modelo linear", segundo o qual o investimento em ciência seria a principal fonte de novos projetos, levando a avanços tecnológicos. Mas o impacto da pesquisa ocorre de maneira bastante diferenciada entre os setores industriais, corroborando os resultados de pesquisas anteriores. Por exemplo, 58\% dos respondentes da indústria farmacêutica apontaram a pesquisa pública como fonte de novos projetos. A pesquisa pública também foi considerada importante para gerar novos projetos nas indústrias de petróleo, aço, maquinários, semicondutores e indústrias aeroespaciais (com 50\% ou mais respondentes).

Narin et al. (1997) fornecem evidências empíricas da crescente importância da ciência pública ${ }^{7}$ para as inovações tecnológicas através da análise das citações das patentes nos Estados Unidos nos períodos 1987-1988 e 1993-1994. Os dados indicam que a ciência pública é responsável por 73,3\% dos artigos citados pelas patentes industriais dos Estados Unidos, ou seja, a maior parte da base científica da indústria americana provém do setor público (Narin et al., 1997, p. 328). ${ }^{8}$ O impacto da ciência na tecnologia também é discutido por Pavitt (1991). Em seu estudo, Pavitt sugere que a influência da ciência na tecnologia não se dá apenas através de avanços no 
conhecimento. Existem outras duas influências tão importantes ou mais: o treinamento em pesquisa e habilidades; e aplicações não planejadas resultantes de conhecimentos adquiridos por meio de pesquisas sem expectativa de aplicações diretas (Pavitt, 1991, p. 114).

Dessa forma, as contribuições da pesquisa acadêmica são importantes para o avanço tecnológico. Contudo, tais contribuições geralmente não se dão de forma direta, e sim indiretamente e através de vários canais. Mowery et al. (2004) destacam que o fluxo de resultados de pesquisas acadêmicas pode ocorrer através de publicações acadêmicas, conferências, palestras, consultorias e migração de profissionais entre a academia e a indústria. Os resultados de Cohen et al. (2002) mostram que os principais canais de transmissão de conhecimento da universidade para a indústria são publicações e consultorias (com 41\% dos respondentes do CMS apontando esse canal como sendo pelo menos moderadamente importante). O estudo de Agrawal e Henderson (2002) descreve os resultados de um survey envolvendo 68 professores dos departamentos de engenharia elétrica e mecânica do MIT, no qual os respondentes apontaram a importância de vários canais de transferência de informação. Os principais canais apontados foram os que os autores chamaram de "informais" (consultoria e conversações, em torno de $31 \%$ ), ficando publicações em segundo lugar, com $18 \%$, e patentes e licenciamentos como os menos importantes (em torno de 7\%).

Embora seja difícil de ser captado por pesquisas, é bastante razoável afirmar que um dos canais mais importantes através do qual os resultados das pesquisas acadêmicas chegam até a indústria seja o conhecimento assimilado pelos estudantes. A universidade moderna passou a agregar, desde o final do século XIX, as atividades de pesquisa e ensino, gerando uma sinergia que propicia aos estudantes terem contato com o conhecimento do estado da arte e com as pesquisas que procuram avançar esse estado. Muitos estudantes, especialmente os da pós-graduação, vão para a indústria com um conhecimento além daquele descrito nos livros-texto, servindo de ponte que leva os avanços científicos para a indústria. Além disso, o conhecimento assimilado pelos estudantes faz com que se tornem peça fundamental para a capacidade de absorção das empresas.

Neste ponto surge a principal diferença entre as universidades e os IPP. Enquanto as universidades combinam pesquisa e ensino em várias áreas do 
conhecimento, os IPP, em geral, são especializados em algumas poucas áreas como ciências agrárias, engenharias e ciências da saúde, e muitos não possuem atividades de ensino. Assim, os canais de fluxo de conhecimentos podem ser ligeiramente diferentes.

Enquanto existe uma vasta literatura sobre a interação universidade-indústria, estudos sobre a interação IPP - indústria são escassos. Um desses trabalhos é o de Goddard e Isabelle (2006), que analisa a relação entre laboratórios públicos de pesquisa e empresas na França. Os autores apontam para a existência de troca de informações entre laboratórios e empresas, gerando uma situação de benefícios mútuos. Comparando os resultados de Goddard e Isabelle com os de Cohen et al. (2002) e Agrawal e Henderson (2002), surgem indicativos de que os canais de transferência de conhecimentos utilizados pelos laboratórios franceses possuem algumas semelhanças com aqueles utilizados pelas universidades dos EUA, com exceção do treinamento de estudantes, o que geralmente não é feito nos laboratórios públicos. Entretanto, este tópico necessita de maiores evidências empíricas, principalmente na comparação entre as dinâmicas de interação das universidades/IPP e a indústria de um mesmo país.

\subsection{Metamorfoses do papel das universidades \\ e IPP no processo de desenvolvimento}

Reconhecendo a relevância da pesquisa acadêmica como fonte de conhecimentos para o avanço tecnológico, governos de vários países industrializados têm buscado incentivar a interação entre universidade e indústria (Mowery et al. 2001; Mowery e Sampat, 2005) e estimular a transferência de conhecimentos tecnológicos da universidade para o setor produtivo, colocando a "comercialização" dos seus produtos como um novo papel para as universidades. Um dos principais marcos que originou essa tendência foi a legislação dos EUA, que, através do Bayh-Dole Act de 1980, facilitou o processo de obtenção de patentes resultantes de pesquisas realizadas com fundos federais e a sua comercialização por parte das universidades. Os proponentes do Bayh-Dole Act argumentaram que os direitos de propriedade intelectual das universidades, licenciados de forma exclusiva, serviriam de estímulos para empresas realizarem os investimentos em pesquisas subseqüentes para gerar uma inovação, contribuindo para a transferência de co- 
nhecimentos tecnológicos para a indústria. De fato, o número de pedidos de patentes das universidades americanas aumentou significativamente ${ }^{9}$ nas décadas de 1980 e 1990. Essa tendência também é observada em países europeus, especialmente a partir da década de 1990, influenciados pela experiência dos EUA (Urraca Ruiz, 2005).

Como a estrutura institucional difere consideravelmente entre os países, o papel das universidades e dos IPP pode variar ${ }^{10}$ e sua influência pode ser potencializada de acordo com a base industrial de uma região, ou país, os incentivos governamentais e de mercado e a capacidade de absorção das empresas. Por exemplo, nos países mais avançados, as universidades têm adquirido o papel de comercializadoras de suas tecnologias, enquanto nos países em desenvolvimento essa tendência ainda é tímida, ${ }^{11}$ embora esteja cada vez mais presente. Dessa forma, papel das universidades e dos IPP pode variar significativamente de acordo com o grau de desenvolvimento econômico e social de um país.

Eun et al. (2006) abordam essas questões de forma bastante elucidativa. Para entender a relação universidade-indústria e o surgimento de empresas geridas por universidades na China, os autores desenvolvem uma estrutura teórica que permite analisar a relação universidade-indústria em países em desenvolvimento. Em 2002, duas das principais empresas fabricantes de computadores pessoais e a principal fabricante de softwares na China eram empresas estabelecidas e geridas por universidades. Além disso, em 2002 existiam 5.047 empresas geridas por universidades, e 14 delas figuravam entre as 100 principais empresas da área de ciência e tecnologia.

Segundo os autores, os fatores determinantes para que uma universidade se torne mais empreendedora e tome parte das funções industriais, estabelecendo empresas e gerindo-as, são: (i) recursos internos da universidade; (ii) capacidade de absorção das empresas e existência de instituições intermediárias; e (iii) propensão das universidades em buscar ganhos econômicos. Dessa maneira, a China acabou por apresentar o surgimento de empresas geridas por universidades devido à conjunção desses fatores. Em primeiro lugar, desde a reforma que buscou tornar o país mais orientado ao mercado, o governo tomou medidas para fazer com que as universidades se tornassem mais voltadas ao desenvolvimento econômico. Uma dessas medidas foi o corte de recursos para as universidades, fazendo-as procurar outras formas 
de financiamento e considerar estabelecer suas próprias empresas, tornando-se mais propensas aos ganhos econômicos. Em segundo lugar, as universidades chinesas possuíam fortes recursos internos, no sentido de possuírem uma experiência em pesquisa aplicada e em desenvolvimento. Além de terem tradição em uma pesquisa aplicada mais forte que a pesquisa básica, sob o regime de planejamento chinês as empresas possuíam apenas uma função na cadeia de produção, que era a manufatura, ficando as outras atividades a cargo dos ministérios. Com freqüência, as universidades tinham de produzir protótipos para as empresas, acumulando experiência para estabelecer suas próprias empresas. Em terceiro lugar, a baixa capacidade de absorção das empresas chinesas constituiu-se em uma barreira à transferência de tecnologia, pois as empresas não tinham capacidade de desenvolver e comercializar os produtos desenvolvidos e oferecidos na forma de licenciamentos pelas universidades. As universidades precisando de recursos, incentivadas pelo governo, tendo experiência na geração de tecnologias e não conseguindo licenciar suas tecnologias, decidiram estabelecer e gerir as suas próprias empresas. Portanto, a atitude das universidades não foi alterada apenas pela redução dos seus recursos financeiros, mas também por um novo "contrato social" que estimulava e endossava essa nova atitude.

Segundo destacam Eun et al. (2006, p. 1.344), esse "modelo" de relação da universidade com o meio econômico não está livre de custos, os quais incluem a redução do tempo destinado à pesquisa e ao ensino, e nem é sustentável a longo prazo. Com o desenvolvimento da capacidade de absorção das empresas, é possível que as universidades passem a se concentrar mais em suas atividades primordiais e possam licenciar suas tecnologias em vez de produzi-las. A estrutura analítica apresentada pelos autores lança luz sobre a questão da relação universidade-indústria em países em desenvolvimento e deixa claro que podem surgir relações diferenciadas dependendo dos fatores envolvidos.

A flexibilidade do sistema de inovação da Coréia do Sul é um exemplo de que o papel das universidades e IPP pode variar ao longo do processo de catching-up. De acordo com Kim (2005), as funções e as formas de interação entre universidades/IPP e empresas foram se alterando conforme o avanço do país. Durante as décadas de 1960 e 1970, no início do processo de catching-up, as universidades concentravam-se no ensino e os IPP realizavam 
P\&D de interesse público e transferiam os resultados para as empresas, ainda deficientes na realização desse tipo de tarefa. Na década seguinte, algumas empresas adquiriram capacidade de realizar P\&D, estabelecendo pesquisas conjuntas com os IPP, que já estavam desenvolvendo um P\&D mais aplicado. Nesse momento, as universidades passaram a se envolver em atividades de pesquisa. O desenvolvimento da capacidade das empresas foi ímpar ao longo dessas décadas. Enquanto 68\% dos gastos com P\&D realizados na Coréia do Sul em 1971 foram feitos pelo governo, os mesmos reduziramse para apenas 20\% em 1987 (Kim, 2005). Na década de 1990, as empresas já possuíam uma forte capacidade de realizar P\&D, ao passo que as universidades começaram a surgir como fontes potenciais de informação para as empresas. Nesse mesmo período, os IPP voltaram-se para pesquisas básicas e para as pequenas empresas.

Portanto, não existe uma única forma de relacionamento entre as universidades e IPP com o setor industrial. Além disso, é importante que exista uma flexibilidade institucional que permita a esses agentes adaptar o seu relacionamento às mudanças econômicas e sociais.

\section{CATCHING-UP: ALGUMAS LIÇõES HISTÓRICAS}

No decorrer dos séculos XIX e XX, alguns países conseguiram avançar tecnologicamente em vários setores industriais, reduzindo a distância com relação aos países líderes e até mesmo ultrapassando-os. O estudo de algumas experiências de sucesso contribui para um melhor entendimento da dinâmica dos mecanismos e agentes que contribuem para o processo de $c a-$ tching-up. Existe uma vasta literatura sobre experiências de sucesso, ${ }^{12}$ sendo o objetivo desta seção buscar características em comum nesses casos de sucesso, em vez de relatá-los.

Mazzoleni e Nelson (2007) sugerem que todos os casos de catching-up de sucesso no passado envolveram três elementos. O primeiro foi um considerável fluxo de pessoas, seja saindo dos países atrasados para receber treinamento nos países mais avançados, seja na forma de consultores provenientes dos países avançados. O segundo elemento foi a ativa participação do governo no processo de catching-up, dando suporte à indústria nascente. O terceiro elemento diz respeito aos direitos de propriedade intelectual. Segundo 
os autores, ao longo do século XIX e grande parte do século XX, os regimes de direito de propriedade intelectual vigentes nos países em desenvolvimento não restringiam seriamente a capacidade das suas empresas imitarem as tecnologias utilizadas nos países avançados. Por exemplo, a maior parte dos países atualmente desenvolvidos concedia patentes para invenções importadas até fins do século XIX (Chang, 2004).

Os exemplos históricos nos permitem acrescentar mais três outros elementos à lista de Mazzoleni e Nelson. ${ }^{13} \mathrm{O}$ primeiro deles relaciona-se ao fato de que, embora a literatura em geral tenha enfatizado o papel do governo na proteção à indústria nascente, o governo também atuou ativamente na promoção da educação, no desenvolvimento do treinamento e da pesquisa aplicada. No século XIX, a Alemanha criou institutos de treinamento e pesquisa que viriam a dar grande suporte à indústria química. No Japão, o governo promoveu a criação de universidades. A Coréia do Sul criou vários institutos de pesquisa, como o Korean Institute of Science and Technology (KIST) e o Korean Institute of Eletronics Technology (KIET), destinado a dar suporte à indústria eletrônica e de semicondutores. Taiwan criou o Industrial Technology Research Institute (ITRI), que contribuiu para o processo de assimilação e difusão de tecnologias consideradas importantes pelo governo, especialmente para o desenvolvimento da indústria de semicondutores (Chen e Sewell, 1996).

Um segundo elemento a ser acrescentado refere-se às inovações organizacionais. Relatando os avanços dos EUA e da Alemanha no final do século XIX e do Japão no início do século XX, Fagerberg e Godinho (2005) destacam que esses países promoveram inovações organizacionais significativas, como o sistema de produção em massa, a organização do P\&D da indústria química e o sistema just-in-time, respectivamente. Os autores acrescentam que

successful catch-up has historically been associated not merely with the adoption of existing techniques in established industries, but also with innovation, particularly of the organization kind, and with inroads into nascent industries (Fagerberg e Godinho, 2005, p. 515).

Por fim, um último elemento a ser acrescentado nessa lista de características observadas nas experiências de catching-up de sucesso está relacionado ao trabalho de Gerschenkron (1962), o qual destaca que as experiências de 
sucesso envolveram, em geral, o investimento nas indústrias mais dinâmicas e progressivas existentes.

Embora Gerschenkron destaque que essa não seja uma condição necessária, havendo países que avançaram sem entrar nas indústrias tecnologicamente mais proeminentes durante o início do processo de catching-up, sua observação é relevante para a análise dos casos mais recentes de catching-up realizados pelos países asiáticos na segunda metade do século XX, como Coréia do Sul e Taiwan. ${ }^{14} \mathrm{O}$ governo japonês também teve, nesse período, um comportamento que ia ao encontro das idéias de Gerschenkron, no sentido de que o MITI (Ministry of International Trade and Industry) via a promoção das mais avançadas tecnologias como uma de suas funções principais, objetivando o mercado mundial (Freeman, 1988, p. 331). Talvez nenhum outro país tenha dado tanta importância à previsão, identificação e exploração de novos paradigmas tecnológicos quanto o Japão.

A idéia de Gerschenkron está estreitamente ligada à de paradigmas tecnológicos, os quais determinam o avanço da fronteira tecnológica e o surgimento de "janelas de oportunidade". Os paradigmas tecnológicos, resultantes de inovações radicais, são determinados pelos países avançados e geram oportunidades tecnológicas devido à sua dinâmica singular. Pérez (2001) apresenta essa dinâmica através da descrição do ciclo de vida do produto e da difusão internacional da tecnologia. A figura 1 apresenta esse processo tendo como exemplo uma inovação tecnológica gerada e produzida inicialmente nos EUA.

Conforme a figura 1, a produção migra inicialmente para outros países desenvolvidos e posteriormente para países menos desenvolvidos. De acordo com Pérez (2001), esse padrão de difusão está associado às mudanças das características das tecnologias em evolução. Uma dessas características refere-se à mão-de-obra. Na fase inicial, as tecnologias são intensivas em mãode-obra mais cara, com elevados níveis de conhecimento e qualificação. Nas fases próximas da maturidade da tecnologia, a produção passa a ser mais intensiva em capital e altamente padronizada, tendendo a utilizar trabalho menos qualificado. É justamente a alteração das características relacionadas à tecnologia que abre espaço para a realização do catching-up. Pérez argumenta que é improvável que ocorra um processo de catching-up baseado em tecnologias já amadurecidas. ${ }^{15}$ Segundo a autora, quando as 
Figura 1: Difusão internacional de tecnologias ao longo do seu processo de amadurecimento

Representação esquemática da posição comercial dos EUA no ciclo de vida do produto

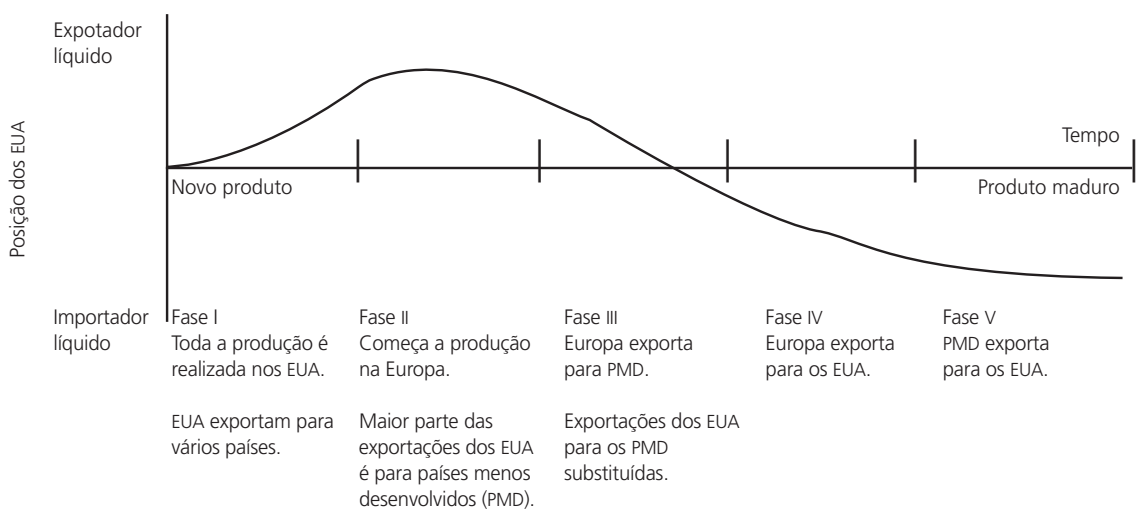

Fonte: Wells (1972, p. 15) apud Pérez (2001, p. 111).

tecnologias alcançam a maturidade, existe pouco espaço para o aumento dos lucros devido à estagnação dos mercados e à redução da possibilidade de aumentos da produtividade (ver últimos três gráficos da figura 2). Assim, embora não seja muito promissor tentar iniciar um processo de catching-up na fase madura, essa fase talvez seja o melhor ponto de partida para iniciar uma base industrial e desenvolver a capacidade de aprendizado crucial para o desenvolvimento (Pérez, 2001, p. 113).

Qual seria então a fase mais promissora para se iniciar o processo de $c a$ tching-up? Pérez argumenta que, excluindo a fase madura, é na fase inicial da tecnologia, na qual existem menores barreiras que podem ser transpostas pelos países menos avançados. ${ }^{16}$ Mas, ao contrário da fase madura, é na fase inicial que existe o maior potencial de realização de lucros. Nesse ponto, fazse a ligação com a observação de Gerschenkron. É na fase inicial do surgimento de novos paradigmas tecnológicos que se abrem "janelas de oportunidade". Contudo, o aproveitamento das oportunidades está condicionado ao desenvolvimento das capacidades de aprendizado e de absorção do país (ver gráficos 1 e 3 da figura 2). Ou seja, os países menos desenvolvidos devem aprender a aproveitar as oportunidades que surgem.

A importância de cada um dos seis elementos discutidos retro relativos às experiências históricas de catching-up varia de acordo com cada caso, e 
Figura 2: Mudanças nas condições de entrada e no potencial das tecnologias ao longo da sua evolução
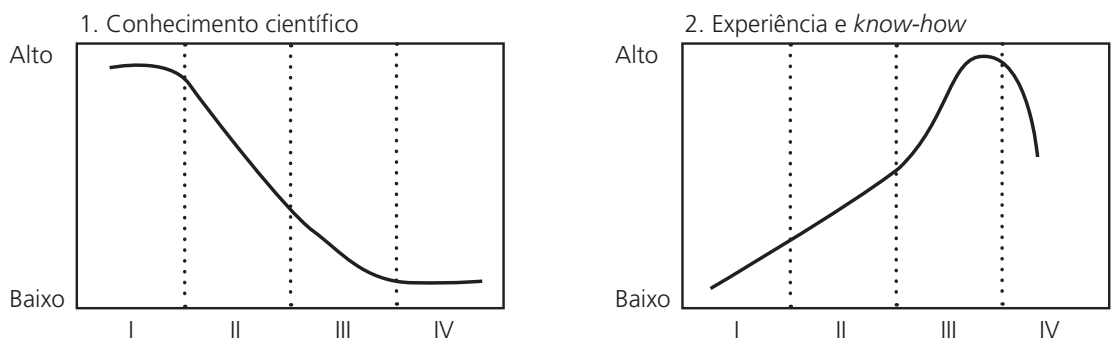

3. Capacidade para usar trabalho não qualificado
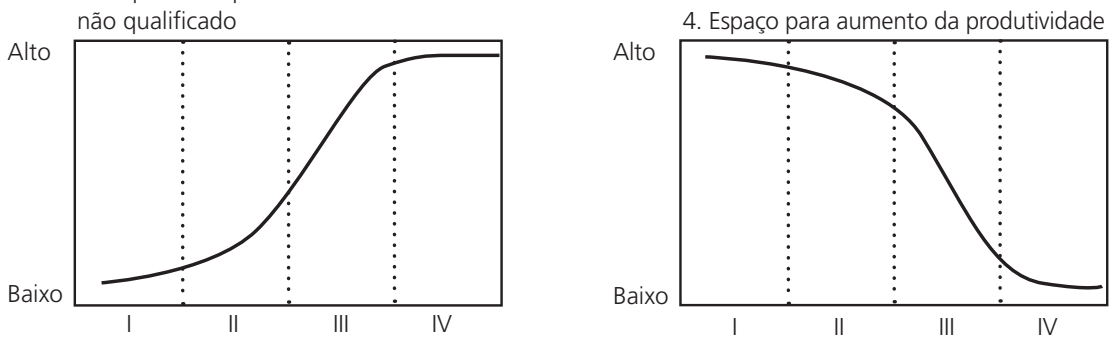

5. Potencial para crescimento do mercado

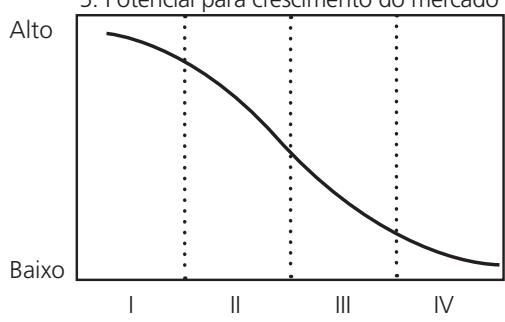

6. Capacidade de realização de lucros

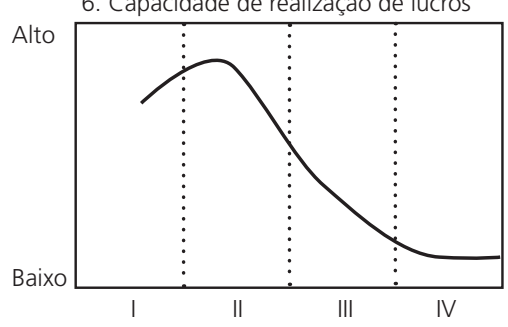


alguns desses elementos tendem a perder importância ao longo do processo de desenvolvimento pelo qual o país passa. Um exemplo disso é a migração de pessoas, que pode ser observada e tem maior importância em etapas preliminares do processo de catching-up. Já outros fatores podem ganhar importância ao longo do processo de desenvolvimento, conforme será discutido adiante.

\section{O PAPEL DAS UNIVERSIDADES E IPP NO PROCESSO DE CATCHING-UP E SUA CRESCENTE IMPORTÂNCIA}

Um dos principais resultados da teoria neoclássica do crescimento econômico trata da tendência de longo prazo à convergência dos níveis de produtividade entre os países. Segundo a hipótese da convergência, a taxa de crescimento da produtividade tende a variar inversamente com o nível de produtividade de um país. Assim, países que estão atrasados tecnologicamente possuem potencial para avançar relativamente mais rápido. $\mathrm{O}$ mecanismo através do qual ocorre a convergência está ligado ao nível tecnológico embutido no estoque de capital do país e à produtividade marginal decrescente do capital. Entretanto, esse mecanismo possui uma autolimitação, pois um país, ao aproximar-se do nível tecnológico dos países líderes, tende a ter o crescimento da sua produtividade desacelerado.

Em um artigo seminal, Abramovitz (1986) reelabora a hipótese neoclássica de convergência, de forma a abarcar a idéia de "capacidades sociais". Para o autor, um país não é tecnologicamente atrasado por mero acidente histórico, mas em grande parte por suas características sociais e culturais. Essas mesmas características podem contribuir para que o atraso permaneça, impedindo a concretização do processo de catching-up que a hipótese neoclássica sugere. ${ }^{17}$ Essa "capacidade social", segundo Abramovitz, é essencial para qualificar a hipótese de convergência, pois, para que um país tenha potencial para um rápido crescimento, não basta ser tecnologicamente atrasado, é preciso que também seja socialmente avançado o suficiente a ponto de poder absorver e explorar as melhores tecnologias existentes.

Contudo, o próprio Abramovitz reconhece que a idéia de capacidade social é difícil de ser conceitualizada e mensurada. Segundo o autor, a capacidade social depende do nível de educação do país e de instituições políti- 
cas, comerciais, industriais e financeiras (Abramovitz, 1986, p. 388-389). A idéia de "socialmente avançado" pode ser, de certa forma, associada a um sistema nacional de inovação desenvolvido o suficiente para absorver os conhecimentos tecnológicos existentes. Fazendo essa associação, a ocorrência do processo de catching-up tecnológico exige que os países tecnologicamente atrasados desenvolvam seus sistemas de inovação promovendo uma melhor conexão entre os agentes e as instituições do sistema, desenvolvendo, ou até mesmo criando, agentes e instituições julgadas necessárias. Isso inclui o desenvolvimento das universidades e IPP.

Além dessa capacidade social, é necessário que os países desenvolvam uma capacidade de absorção de conhecimentos tecnológicos. Cohen e Levinthal $(1989,1990)$ desenvolvem a idéia de capacidade de absorção como sendo a capacidade da empresa de reconhecer, assimilar e explorar comercialmente novas idéias. Os autores sugerem que a atividade de P\&D não gera apenas um produto (nova informação), como os economistas costumavam pensar, mas gera também a capacidade da empresa de "assimilar e explorar as informações existentes".

A idéia de capacidade de absorção pode ser utilizada para entender os países em seu processo de catching-up e o papel das universidades e IPP. Os estudos sobre as experiências mais recentes têm destacado que a importância da pesquisa científica realizada nas universidades e IPP para o processo de catching-up tecnológico tem sido crescente (Albuquerque, 2001; Mazzoleni, 2003; Mazzoleni e Nelson, 2007; Nelson, 2004; Rapini et al. 2006). Albuquerque (2001), analisando a infra-estrutura científica de 17 países e tomando os casos da Coréia do Sul e Taiwan como referência, confirma que um certo nível interno de conhecimento científico não é condição suficiente, mas se faz cada vez mais necessário para melhorar a capacidade de absorção do país e para iniciar o processo de catching-up. De acordo com Nelson (2004, p. 355), "the process of catch-up essentially involves learning about and learning to master ways of doing things that are used by the leading countries of the era". Assim, as pesquisas científicas nas universidades e IPP possuem papel importante no processo de catching-up tecnológico por contribuírem para o aprendizado das tecnologias existentes.

A crescente importância do papel das universidades e IPP no processo de catching-up fica evidenciada pelo crescente aumento da base científica dos 
novos paradigmas tecnológicos. Contudo, não se espera que os países em desenvolvimento tenham de elevar suas pesquisas científicas ao mesmo patamar dos países avançados para realizar o catching-up. Países que são líderes em vários ramos tecnológicos, como Japão e Coréia do Sul, não são necessariamente líderes em pesquisas relacionadas a esses ramos.

Rapini et al. (2006, p. 4) apontam que as pesquisas realizadas nas universidades e institutos de pesquisa, além de contribuírem para o aumento da capacidade de absorção, possuem outras importantes funções no processo de desenvolvimento tecnológico. Primeiro, servem como um "instrumento de focalização", estabelecendo vínculos com fontes internacionais de tecnologia, contribuindo para avaliar quais desenvolvimentos tecnológicos estão disponíveis e apontar em quais setores industriais a entrada é difícil. Segundo, a pesquisa científica serve como suporte para a indústria nacional, permitindo-a entrar em indústrias importantes para o desenvolvimento. Portanto, o papel da ciência nos países tecnologicamente atrasados difere daquele exercido em países avançados. Nos países tecnologicamente avançados, os desenvolvimentos científicos são considerados uma das principais fontes de oportunidades tecnológicas (Klevorick et al., 1995). Já nos países atrasados, o papel da pesquisa científica está mais em auxiliar na identificação das oportunidades tecnológicas geradas no exterior (Albuquerque, 2001, p. 548). Ou seja, embora os conhecimentos científicos tenham características especiais que permitem qualificá-los como bens públicos (Arrow, 1962; Nelson, 1959), não se pode deixar de reconhecer que, para se tornarem bens de livre acesso, é preciso que haja capacidade de entender, interpretar e, por fim, absorver os conhecimentos científicos e tecnológicos "disponíveis”. E isso exige que o país desenvolva a sua capacidade de pesquisa.

Moravcsik (1983) apresenta uma análise do papel da ciência no processo de transferência internacional de tecnologia e conclui que existe uma necessidade de estimular e investir nas atividades científicas de um país o mais cedo possível. Para o autor, a produção interna de ciência é importante como forma de aprender, assimilar e imitar, mesmo encarando a transferência de tecnologia como sendo a importação de produtos tecnológicos.

Além da influência direta para o catching-up tecnológico, a pesquisa científica contribui para o desenvolvimento econômico de outras formas. Mazzoleni e Nelson (2007) destacam que a pesquisa científica contribui 
para avanços em setores em que a simples importação de tecnologia não é eficiente, pois necessita de ajustamentos para se adaptar ao novo ambiente, especialmente nas áreas da agricultura e saúde. Esses ajustamentos freqüentemente levam a avanços no entendimento das especificidades do país e a melhorias tecnológicas.

Contudo, para que a pesquisa realizada nas universidades e IPP possa contribuir para o catching-up tecnológico, é necessário que haja um diálogo e um estreitamento das suas relações com as empresas. Esse diálogo é fundamental, pois as empresas de países em desenvolvimento possuem pouca capacidade de absorver os conhecimentos gerados nas universidades e IPP. Essa baixa capacidade está associada à tendência de as empresas desses países usarem tecnologias maduras, em que há pouca necessidade de se engajar em atividades de P\&D (Pérez, 2001). O caso da Coréia do Sul ilustra bem essa situação. Enquanto o seu desenvolvimento baseou-se em tecnologias maduras, as empresas engajaram-se em poucas atividades de P\&D. Nessa fase, os IPP coreanos desempenharam a importante tarefa de ajudar as empresas a adquirirem e assimilarem as tecnologias estrangeiras (Kim, 2005). Quando as empresas passaram a realizar P\&D sistematicamente, puderam contratar pesquisadores dos IPP que haviam adquirido experiência em engenharia reversa nos laboratórios públicos nas décadas anteriores. Kim (2005, p. 303) destaca que:

(...) os institutos públicos de pesquisa não devem ser analisados pelo número de patentes ou de pesquisas importantes que geram e transferem ao setor privado, mas pelo número de pesquisadores experientes que formam, indivíduos capazes de exercer papéis de liderança nas atividades de P\&D industrial do setor privado.

Ainda são poucos os estudos sobre a interação universidade-indústria nos países em desenvolvimento. Um desses estudos avalia essa interação no Brasil, mais especificamente no Estado de Minas Gerais, através de uma pesquisa com 140 empresas que realizam P\&D (Rapini et al., 2006). Os autores sugerem que, devido ao fraco envolvimento das empresas em atividades de P\&D em sistemas de inovação imaturos, as universidades, ao se relacionarem com a indústria, podem ter um papel dual, tanto complementando o P\&D industrial quanto o substituindo. Essa dualidade é reflexo, dentre outros fatores, da distribuição dos pesquisadores entre a indústria, as universi- 
dades e os IPP. Enquanto nos países desenvolvidos grande parte dos pesquisadores encontra-se na indústria (Mowery e Sampat, 2005), nos países em desenvolvimento a maior parte está nas universidades e IPP. Isso faz com que a indústria nos países em desenvolvimento não tenha a mesma capacidade de absorção que nos países desenvolvidos, afetando alguns canais de transferência de conhecimentos, principalmente da universidade para a indústria. Conforme discutido anteriormente, parte dos avanços científicos chega à indústria embutida no treinamento recebido pelos estudantes, em especial os estudantes de pós-graduação. Contudo, esse fluxo de conhecimentos é fraco nos países em desenvolvimento, permanecendo a maior parte dos pós-graduados na carreira acadêmica e no setor público.

\section{CATCHING-UP: NOVOS DESAFIOS}

Considerando as mudanças recentes relacionadas às regras do comércio internacional (principalmente os resultados da Rodada Uruguai do GATT) e aos novos paradigmas tecnológicos, é válido questionar se está ficando mais difícil para os países tecnologicamente atrasados realizar um processo de catching-up.

Dadas as lições históricas discutidas anteriormente, podemos dizer que em alguns aspectos está ficando mais difícil alcançar os países líderes em tecnologia. Dos seis elementos característicos apresentados na segunda seção, pelo menos três estão mais difíceis de serem colocados em prática pelos países mais atrasados.

Em primeiro lugar, as principais instituições internacionais, como o Banco Mundial, a Organização Mundial do Comércio e o Fundo Monetário Internacional, desestimulam com veemência a atuação dos governos de países em desenvolvimento na proteção da indústria nascente, ficando estes impedidos de utilizar mecanismos amplamente usados por países agora desenvolvidos para proteger a sua indústria (Chang, 2004).

Em segundo lugar, como destacado em Mazzoleni e Nelson (2007), o mundo do século XXI apresenta características tais como um ambiente legal internacional mais rígido, em que as empresas de países mais desenvolvidos estão mais agressivas na proteção dos seus direitos de propriedade intelectual. Segundo Albuquerque (1998, p. 203-204), “há uma clara tensão entre o crescimento do peso da informação e do conhecimento na dinâmica econô- 
mica contemporânea e as tentativas de bloquear ou dificultar a sua difusão". Um desses indicativos foi a pressão exercida pelos EUA sobre os países em desenvolvimento pela adesão ao acordo TRIPS (Trade Related Intellectual Property Rights). O acordo TRIPS, resultante (entre outros fatores) da busca dos EUA de ligar as questões de propriedade intelectual com as do comércio internacional, resultou em um "fortalecimento das 'barreiras artificiais' à livre difusão e ao livre acesso de um estoque crescente de conhecimento codificado" (Albuquerque, 1998, p. 166). Assim, a imitação das tecnologias dos países avançados, uma das estratégias usadas pelos países que realizaram catching-up, está ficando cada vez mais difícil de ser utilizada sem que os países atrasados invistam em pesquisas e treinamento para aprender essas tecnologias e imitá-las sem infringir os direitos de propriedade.

A importância das universidades e IPP para o desenvolvimento de conhecimentos e soluções em certas áreas tecnológicas em que a proteção intelectual é fundamental é considerável. No Brasil, por exemplo, mesmo realizando em torno apenas de 1,6\% dos depósitos de patentes de residentes entre 1998 e 2001, as universidades e IPP brasileiros foram responsáveis por $56,3 \%$ dos depósitos na área de biotecnologia e 45\% em química orgânica (Póvoa, 2008).

Por fim, uma das características dos países que realizaram o catching-up foi o estímulo à entrada nas indústrias com as tecnologias mais modernas. Entretanto, as tecnologias mais avançadas estão cada vez mais baseadas na ciência, fazendo com que o treinamento avançado e certo nível de pesquisas científicas sejam condições necessárias para absorver os conhecimentos tecnológicos. Para os países que pretendem realizar o catching-up, torna-se cada vez mais importante a atuação das universidades e IPP tanto no treinamento quanto na pesquisa científica para desenvolver capacidades de identificar e entrar nas áreas tecnológicas mais avançadas.

\section{COMENTÁRIOS FINAIS}

Este artigo teve como objetivo analisar o papel das universidades e dos institutos públicos de pesquisa no processo de avanço tecnológico de países em desenvolvimento. Foram apresentados argumentos que sugerem que a importância desses agentes para o avanço tecnológico tem aumentado. 
Ao revisar as características dos processos de catching-up do passado, identificou-se como um importante elemento a entrada nas mais dinâmicas indústrias. Diante dos novos paradigmas tecnológicos, cada vez mais baseados na ciência, a pesquisa realizada nas universidades e institutos de pesquisa acaba por se tornar fundamental para identificar essas novas tecnologias e aprendê-las, contribuindo para difundi-las, através da função de treinamento avançado, para o restante da economia.

Dentre os novos desafios a serem superados está o desenvolvimento da capacidade de gerar soluções tecnológicas diante do fortalecimento da proteção à propriedade intelectual no cenário internacional decorrente do acordo TRIPS. Essa capacidade está estreitamente ligada à pesquisa desenvolvida nas universidades e institutos de pesquisa de um país.

Ao se observar o processo de catching-up do Japão, nota-se que uma das principais características do seu sistema de inovação é a preocupação em prever novos paradigmas tecnológicos e a capacidade de difundir essas expectativas para o maior número possível de empresas nas mais variadas indústrias (Freeman, 1988). Nesse contexto fica evidenciada a crescente importância das universidades e IPP dentro do sistema nacional de inovação. A pesquisa acadêmica pode ajudar no monitoramento e na previsão dos novos paradigmas, e a interação com a indústria é importante desde o início do processo de desenvolvimento, pois serve como apoio para difundir essas expectativas para as empresas.

\section{NOTAS}

1. O conceito de sistema nacional de inovação apresenta uma história interessante. Além de os professores Freeman e Lundvall atribuírem um ao outro a primeira referência ao termo (ver Albuquerque, 2004), o mesmo passou a ser utilizado simultaneamente por vários pesquisadores com significados um pouco distintos (Freeman, 1988; Freeman, 1995; Lundvall, 1992; Nelson e Rosenberg, 1993). Para Edquist (2005), Lundvall considera a "estrutura produtiva" e o "arranjo institucional" como sendo as dimensões cruciais do sistema de inovação, enquanto Nelson e Rosenberg enfatizam as organizações que geram e difundem o conhecimento. Edquist também destaca que não há um consenso sobre quais organizações e instituições devem ser consideradas como parte de um sistema de inovação e quais devem ser excluídas (nesse ponto, trabalhos empíricos podem ajudar a delinear as "fronteiras do sistema").

2. Alguns autores chegam a tratar também de questões regionais aplicando esse conceito. Para mais detalhes sobre sistemas regionais de inovação, ver Asheim e Gertler (2005). 
3. Embora a maior parte da literatura se concentre na relação entre a universidade e a indústria, iremos considerar também a pesquisa realizada nos IPP e sua relação com a indústria. Não obstante, veremos a seguir que existem algumas diferenças entre o papel da universidade e o dos IPP.

4. As oportunidades tecnológicas compreendem o "conjunto de possibilidades para o avanço tecnológico" (Klevorick et al., 1995, p. 188).

5. Para mais detalhes e as diferenças entre o CMS e o YS, ver Cohen et al. (2002, p. 12).

6. O estudo de Gibbons e Johnston (1974) aponta para uma conclusão semelhante, colocando mais ênfase ainda no papel dos cientistas acadêmicos na resolução de problemas de projetos em andamento.

7. Para os autores, "ciência pública" é a pesquisa científica realizada não só nas universidades, como também nas instituições de pesquisa governamentais e financiadas por agências governamentais e filantrópicas.

8. Uma importante crítica a essa abordagem é feita por Pavitt (1991), em que o autor ressalta que parte da tecnologia gerada não é patenteada e essa tecnologia pode não ser tão dependente da ciência, e sim das habilidades em engenharia e do "design cumulativo".

9. Análises do aumento das patentes de universidades do EUA estão presentes nos estudos de Henderson et al. (1998) e de Mowery et al. (2001).

10 . Ver Nelson (1988) para o caso dos EUA e Freeman (1988) para o caso do Japão.

11. Devido a limitações das universidades e de sua indústria.

12. Ver a revisão apresentada por Fagerberg e Godinho (2005).

13. Claramente, todos esses elementos estão entrelaçados, sendo difícil afirmar quais foram os mais importantes em cada caso.

14. Embora o início da industrialização na Coréia e Taiwan tenha sido baseado na "imitação" de tecnologias maduras, esses países procuraram entrar nas indústrias tecnologicamente mais avançadas logo que desenvolveram uma capacidade de aprendizado.

15. Embora seja improvável, não é impossível. Um novo paradigma tecnológico pode rejuvenescer indústrias maduras, trazendo ganhos de produtividade e novas formas organizacionais, tornando-as dinâmicas novamente. Um momento especial para que isso ocorra é durante a transição de paradigmas (Pérez, 2001, p. 117-119).

16. Na fase inicial, tende a existir uma livre competição entre as firmas para ganhar aceitação no mercado, leva-se um certo tempo até ser estabelecido o design dominante do produto e as exigências em termos de experiência e know-how ainda são baixas.

17. Enquanto os estudos sobre convergência baseiam-se na teoria neoclássica do crescimento, que prevê uma convergência dos níveis de produtividade dos países, os estudos sobre o processo de catching-up têm abordado, em geral, as condições sob as quais certos países conseguiram avançar, procurando identificar políticas a serem adotadas para desencadear um processo de catching-up. Assim, para haver convergência é preciso que os países atrasados realizem o catching-up. 


\section{REFERÊNCIAS BIBLIOGRÁFICAS}

ABRAMOVITZ, M. Catching up, forging ahead, and falling behind. Journal of Economic History, v. 66, n. 2, p. 385-406, jun. 1986.

AGRAWAL, A.; HENDERSON, R. Putting patents in context: exploiting knowledge transfer from MIT. Management Science, v. 48, n. 1, p. 44-60, jan. 2002.

ALBUQUERQUE, E. Scientific infrastructure and catching-up process: notes about a relationship illustrated by science and technology statistics. Revista Brasileira de Economia, v. 55 , n. 4 , p. $545-566,2001$.

Apresentação do artigo The national system of innovation in historical perspective. Revista Brasileira de Inovação, Rio de Janeiro, v. 3, n. 1, p. 9-13, 2004.

Patentes de invenção de residentes no Brasil (1980-1995): uma investigação sobre a contribuição dos direitos de propriedade intelectual para a construção de um sistema nacional de inovação. 275 f. (Tese de Doutorado em Economia) - Instituto de Economia, Universidade Federal do Rio de Janeiro, 1998.

ARROW, K. Economic welfare and the allocation of resources for invention. In: NELSON, R. (Ed.). The rate and direction of inventive activity. Princeton, NJ: Princeton University, 1962, p. 609-625.

ASHEIM, B.; GERTLER, M. The geography of innovation: regional innovation systems. In: FAGERBERG, J.; MOWERY, D.; NELSON, R. (Eds.). The Oxford handbook of innovation. Oxford: Oxford University, 2005, p. 291-317.

CHANG, H-J. Chutando a escada: a estratégia do desenvolvimento em perspectiva histórica. São Paulo: Unesp, 2004, 266 p.

CHEN, C-F.; SEWELL, G. Strategies for technological development in South Korea and Taiwan: the case of semiconductors. Research Policy, v. 25, n. 5, p. 759-783, 1996.

COHEN, W.; LEVINTHAL, D. Innovation and learning: the two faces of R\&D. The Economic Journal, v. 99, n. 397, p. 569-596, 1989.

Absorptive capacity: a new perspective on learning and innovation. Administrative Science Quarterly, v. 35, p. 128-152, 1990.

— NELSON, R.; WALSH, J. Links and impacts: the influence of public research on industrial R\&D. Management Science, v. 48, n. 1, p. 1-23, jan. 2002.

DOSI, G.; FREEMAN, C.; NELSON, R.; SILVERBERG, G.; SOETE, L. (Eds.). Technical change an economic theory. Londres: Printer, 1988, 646 p.

EDQUIST, C. Systems of innovation: perspectives and challenges. In: FAGERBERG, J; MOWERY, D.; NELSON, R. (Eds.). The Oxford handbook of innovation. Oxford: Oxford University, 2005, p. 181-208.

EUN, J-H.; LEE, K.; WU, G. Explaining the "university-run enterprises" in China: a theoretical framework for university-industry relationship in developing countries and its application to China. Research Policy, v. 35, n. 9, p. 1.329-1.346, nov. 2006.

FAGERBERG, J.; GODINHO, M. Innovation and catching-up. In: FAGERBERG, J.; MOWERY, D.; NELSON, R. (Eds.). The Oxford handbook of innovation. Oxford: Oxford University, 2005, p. 291-317. 
; MOWERY, D.; NELSON, R. (Eds.). The Oxford handbook of innovation. Oxford: Oxford University, 2005, $686 \mathrm{p}$.

FREEMAN, C. Japan: a new national system of innovation? In: DOSI, G.; FREEMAN, C.; NELSON, R.; SILVERBERG, G.; SOETE, L. (Eds.). Technical change an economic theory. Londres: Printer, 1988, p. 330-348.

The "National System of Innovation" in historical perspective. Cambridge Journal of Economics, v. 19, n. 1, p. 5-24, 1995.

GERSCHENKRON, A. Economic backwardness in historical perspective: a book of essays. Cambridge, Mass: Belknap, 1962, 456 p.

GIBBONS, M.; JOHNSTON, R. The roles of science in technological innovation. Research Policy, v. 3, n. 3, p. 220-242, nov. 1974.

GODDARD, J.; ISABELLE, M. How do public laboratories collaborate with industry? New survey evidence from France. Paris: Université Paris-Dauphine/Institut pour le Management de la Recherche et de l'Innovation (IMRI), 21 p. (Working papers; 0602), 2006.

HENDERSON, R.; JAFFE, A.; TRAJTENBERG, M. Universities as a source of commercial technology: a detailed analysis of university patenting, 1965-1988. The Review of Economic and Statistics, v. 80, n. 1, p. 119-127, 1998.

JAFFE, A. B. Real effects of academic research. American Economic Review, v. 79, n. 5, p. $957-$ 970, dec. 1989.

KIM, L. Da imitação à inovação: a dinâmica do aprendizado tecnológico da Coréia. Campinas, SP: Unicamp, 2005, 388 p. (Clássicos da inovação).

KLEVORICK, A.; LEVIN, R.; NELSON, R.; WINTER, S. On the sources and significance of interindustry differences in technological opportunities. Research Policy, v. 24, n. 2, p. 185-205, mar. 1995.

LEE, K.; LIM, C. Technological regimes, catching-up and leapfrogging: findings from the Korean industries. Research Policy, v. 30, n. 3, p. 459-483, mar. 2001.

LEVIN, R.; KLEVORICK, A.; NELSON, R.; WINTER, S. Appropriating the returns from industrial research and development. Brookings Papers on Economic Activity, n. 3, Special Issue on Microeconomics, p. 783-831, 1987.

LUNDVALL, B. National systems of innovation: towards a theory of innovation and interactive learning. Londres: Pinter, 1992, 342 p.

MANSFIELD, E. Academic research underlying industrial innovations: sources, characteristics, and financing. The review of Economics and Statistics, v. 77, n. 1, p. 55-65, feb. 1995.

MAZZOLENI, R. The role of universities and public research in catching up process. Disponível em: <http://redesist.ie.ufrj.br/globelics/pdfs/GLOBELICS_0057_Mazzoleni.PDF> 2003.

; NELSON, R. Public research institutions and economic catch-up. Research Policy, v. 36, n. 10, p. 1.512-1.528, dec. 2007.

MEYER-KRAHMER, F.; SCHMOCH, U. Science-based technologies: university-industry interactions in four fields. Research Policy, v. 27, n. 8, p. 835-851, dez. 1998.

MORAVCSIK, M. The role of science in technology transfer. Research Policy, v. 12, n. 5, p. $287-$ 296, out. 1983. 
MOWERY, D.; NELSON, R.; SAMPAT, B.; ZIEDONIS, A. The growth of patenting and licensing by U.S. universities: an assessment of the effects of the Bayh-Dole act of 1980. Research Policy, v. 30, n. 1, p. 99-119, jan. 2001.

Ivory Tower and industrial innovation: university-industry technology transfer before and after the Bayh-Dole act in the United States. Stanford, CA.: Stanford Business Books, 2004, $241 \mathrm{p}$.

; ROSENBERG, N. Trajetórias da inovação: a mudança tecnológica nos Estados Unidos da América no século XX. Campinas, SP: Unicamp, 2005, 230 p. (Clássicos da inovação).

—__ SAMPAT, B. Universities in national innovation systems. In: FAGERBERG, J.; MOWERY, D.; NELSON, R. (Eds.). The Oxford handbook of innovation. Oxford: Oxford University, 2005, p. 209-239.

NARIN, F.; HAMILTON, K.S.; OLIVASTRO, D. The increasing linkage between US. technology and public science. Research Policy, v. 26, n. 3, p. 317-330, out. 1997.

NELSON, R. R. The simple economics of basic scientific research. Journal of Political Economy, v. 67, n. 3, p. 297-306, 1959.

The market economy, and the scientific commons. Research Policy, v. 33, n. 3, p. 455-471, abr. 2004.

Institutions supporting technical change in the United States. In: DOSI, G.; FREEMAN, C.; NELSON R.; SILVERBERG, G.; SOETE, L. (Eds.). Technical change an economic theory. Londres: Printer, 1988, p. 312-329.

ROSENBERG, N. Technical innovation and national systems. In: NELSON R. (Ed.). National innovation systems: a comparative analysis. New York: Oxford University, 1993, p. 3-21.

PAVITT, K. What makes basic research economically useful? Research Policy, v. 20, n. 2, p. 109119, abr. 1991.

PÉREZ, C. Technological change and opportunities for development as a moving target. Cepal Review, n. 75, p. 109-130, dez. 2001.

PÓVOA, L. M. C. Patentes de universidades e institutos públicos de pesquisa e a transferência de tecnologia para empresas no Brasil. 153 f. (Tese de Doutorado em Economia) - Cedeplar, Universidade Federal de Minas Gerais, 2008.

RAPINI, M.; ALBUQUERQUE, E. M.; SILVA, L.; SOUZA, S.; RIGHI, H.; CRUZ, W. Spots of interaction: an investigation on the relationship between firms and universities in Minas Gerais. Belo Horizonte: UFMG/Cedeplar, 2006, 47 p. (Texto para Discussão n. 286).

ROSENBERG, N. Inside the black box: technology and economics. Cambridge: Cambridge University, 1982, $304 \mathrm{p}$.

Schumpeter and the endogeneity of technology: some American perspectives. Library Binding, Routledge, 2000, 142 p.

; NELSON, R. American universities and technical advance in industry. Research Policy, v. 23, n. 3, p. 323-348, 1994.

URRACA RUIZ, A. Patentes y función publica universitaria en Europa: mitos y realidades. Revista Brasileira de Inovação, v. 4, n. 2, p. 391-423, 2005. 